\title{
Business-Intelligence-gestützte Vorauskalkulation in der Primärgewinnungsindustrie
}

\author{
Johannes Buder, Carsten Felden, Joel Sieber \\ Professur für ABWL, insh. für Informationsmanagement/Wirtschaftsinformatik, \\ Technische Universität Bergakademie Freiberg
}

\section{Einleitung}

Die europäische Braunkohleindustrie ist seit Anfang der achtziger Jahre mit rückläufigen oder stagnierenden Absatzvolumina konfrontiert. Die damit einhergehende kontinuierliche Margenerosion zwingt die Industrie zu permanenten Kostenreduzierungen. Die Braunkohlenbergbaue sind von dieser Entwicklung besonders betroffen (Konstantin 2007, S. 52). Bei stetig steigendem Wettbewerbsdruck und sinkenden Deckungsbeiträgen aus der Weiterverarbeitung ist die Tagebauplanung ein wesentlicher Wettbewerbsvorteil, um dem hohen Fixkostenblock begegnen zu können und Kostensenkungspotenziale im Gesamtprozess zu schaffen. Daher erwächst aus diesen Anforderungen bei den Braunkohlegesellschaften der Handlungsbedarf, den Planungsprozess auf Basis verlässlicher und konsistenter Daten durchzuführen. Bis dato wurde in der Literatur nicht untersucht, ob Konzepte der Business-Intelligence, die in anderen Branchen in diesem Kontext diskutiert werden, hier eine adäquaten Lösungsansatz bieten. Es ist daher das Ziel des Beitrages, ausgehend von einem vorhandenen Fachkonzept zur Vorauskalkulation von Investitionsprojekten im Bergbau, ein multidimensionales Datenmodell zu entwerfen, welches den Vorausplanungsprozess im Tagebau effektiv und effizient unterstützt und damit einen Lösungsansatz als Business-Intelligence-Lösung zur Prozesstransparenz und Kostenreduktion begründet.

Die in der Praxis übliche Verwendung von Tabellenkalkulationsprogrammen wird den genannten Anforderungen nicht gerecht. Diese basieren zwar auch auf den fachlichen Konzepten zur Bewertung von Lagerstätten (Wellmer et al. 2008, S. 63f), weisen aber in Bezug auf eine effiziente und effektive Bewertung eine Reihe von Nachteilen auf. Zum einen erfolgt die Datenkonsolidierung der Quellsysteme manuell, so dass die Validität der Daten beeinträchtigt wird. Zum anderen müssen für die Kennzahlen sowohl betriebswirtschaftliche als auch technische und geologische Daten konsolidiert werden. Diese Problemstellung ist aus informationstechnischer Sicht bisher nicht untersucht worden aber wichtiger Bestandteil einer umfassenden Lösung. Die Verwendung von Tabellenkalkulationsprogram- 
men kann zu einem unübersichtlichen Dateisystemansatz führen, der den Anforderungen an Validität und Konsistenz nicht entspricht und in den 1990er Jahren als Basis der Business-Intelligence-Diskussion in Unternehmen diente (Totok und Jaworski 1998, S. 33). Ferner erfolgt keine Erfassung der Planungsergebnisse und Schätzfaktoren über den Zeitverlauf. Die fehlende Erfassung wirkt sich in einer geringeren Verlässlichkeit bei einem Vergleich der Planszenarios und der Planungsergebnisse aus. In diesem Kontext bieten Business Intelligence (BI) Systeme die Möglichkeit, das bisher implizite Wissen in Form von Schätzparametern systematisch zu erfassen. Ferner ist BI ein Konzept, das zu den exogenen Schätzparametern die Leistungsdaten erfassen kann, um so den Anforderungen der Kostenkontrolle im Vorauskalkulationsprozess zu entsprechen. Bieten bestehende Ansätze (Wellmer et al. 2008) eine ausschließlich mengenorientierte Betrachtung, wird in diesem Beitrag aufgezeigt, dass eine Verwendung multidimensionaler Datenstrukturen ein Kennzahlensystem ermöglicht, der die von Slaby und Wilke (2006) entwickelte wertgrößenorientierte Prozesssicht zulässt, um eine effektive Kostenkontrolle zu ermöglichen.

Um das Ziel eines multidimensionalen Datenmodells zu erreichen, erfolgt zunächst eine Einführung in die Grundlagen der Investitionsplanung im Tagebau sowie die Darstellung des Status Quo des Vorauskalkulationsprozesses (Kapitel 2). Anschließend wird im Kapitel 3 das Datenmodell im Kontext des analytischen Datenbanksystems aufgezeigt, ehe im Kapitel 4 die Erläuterung der theoretische Rahmen und des gewählten Vorgehens erfolgt. Das Kapitel 5 erklärt zunächst das fachliche Konzept zur Investitionsplanung im Braunkohlebergbau, ehe das logische und semantische Datenmodell als Ergebnisartefakte dargestellt wird. Im Kapitel 6 erfolgt eine Zusammenfassung und Beurteilung der Ergebnisse.

\section{Investitionsplanung im Tagebau}

Der Bergbau grenzt sich anhand der elementaren Produktionsfaktoren von anderen Industrien dahingehend ab, als dass eine gegebene, nicht reproduzierbare Rohstofflagerstätte die substanzielle Grundlage eines Bergbaubetriebes ist. Die Ergebnisse der technischen Gutachten sind Grundlage einer betriebswirtschaftlichen Bewertung, die als Bauwürdigkeitsprüfung bezeichnet wird (Haase und Pfennig 2009a, 59). In diesem Kontext sind die Quantität, die Qualität und die Bonität des Rohstoffs wichtige Faktoren (Slaby und Wilke 2005, S. 11). Die Quantität beschreibt die effektiv nutzbare Vorratsmenge, während die Qualität den Wertstoffgehalt misst. Die Bonität beurteilt die Lagerungsbedingungen (Fettweis 1990, S. 29). Rohstoffvorkommen sind dann bauwürdig, wenn zum Zeitpunkt der Bewertung nachweisbar ist, dass der abzubauende Rohstoff über einen definierten Zeitraum mit wirtschaftlichem Erfolg gewonnen, verarbeitet und veräußert werden kann. Die Bauwürdigkeitsuntersuchung ist die zentrale Bewertungsgrundlage der Investitionsentscheidung, welche im Rahmen der Liberalisierung der Energiemärk- 
te zunehmend in den Fokus rückt. In diesem Kontext gewinnen zugleich Kostenkontrolle und Kostentransparenz mit dem Ziel der Kostenreduktion an Bedeutung (Felden 2008, S. 84).

Die betriebswirtschaftliche Bewertung einer Investitionsentscheidung im Tagebau ist aufgrund ihrer Irreversibilität von besonderer Bedeutung (Slaby und Wilke 218, S. 221). Zwar existieren bereits Ansätze, welche die Qualität der Lagerstätten sowie die Erlös-, Kapazitäts- und Betriebskostenkalkulation auf Basis von Tabellenkalkulationsprogrammen abbilden (Wellmer et al. 2008, S. 63ff), jedoch ist deren Einsatz besonders bei hohen Datenvolumina problematisch, da getrennt Daten über geologische Lagerstätten sowie technische und betriebswirtschaftliche Artefakte erfasst werden. In Hinblick auf die Datenkonsolidierung zeigt sich ein begrenztes Funktionsspektrum der Tabellenkalkulationsprogramme, so dass die Einbindung der verschiedenen Datenquellen manuell erfolgt und daher fehleranfällig ist (Gluchowski et al. 2008, 102). Ferner ist ein solcher Dateisystemansatz nicht geeignet, Anforderungen an eine einheitliche Versionierung der Daten, Authentifizierung und Autorisierung zu entsprechen. Ein schwerwiegender Nachteil stellt die fehlende Dokumentation und Erschließung der Ergebnisse der historischen Planung und Produktion dar (Felden 2008, S. 85). Die Vorausplanung ist von einer Vielzahl an sich über den Zeitverlauf ändernden Schätzparametern abhängig, welche in ihrer Historie nicht erfasst werden. Daher bleiben gewonnene Erkenntnisse implizit, so dass ein Soll/Ist- bzw. Soll/Soll-Vergleich im Rahmen einer Sensitivitätsanalyse erschwert wird. Die fehlende Erschließung bewirkt eine Informationslücke zwischen langfristig orientierten Investitionsentscheidungen im Bergbau und den dazu notwendigen Informationen aus der operativen Geschäftstätigkeit (Kemper et al. 2006, S. 63). Der Status Quo begründet daher den Aufbau eines Data Warehouse im Rahmen des BI-Konzepts als Integrationspunkt, da bestehende Ansätze der Problemlösung im Rahmen einer effektiven und effizienten Planung nicht gerecht werden.

\section{Modellierung des Data Warehouse}

Aus den dargestellten Nachteilen des bisherigen Vorausplanungsprozesses erwachsen die Anforderungen, Daten automatisch zu konsolidieren, die für die Investitionsentscheidung relevanten Kennzahlen zu berechnen und die Ergebnisse der Planung zu speichern. Business Intelligence (BI) Systeme erfüllen diese Anforderungen. Der Begriff BI umfasst alle Anwendungen von entscheidungsorientierten Informationssystemen, von der Sammlung über die Speicherung und Aufbereitung der Daten und ihre Präsentation. Er ist losgelöst von technischen Artefakten (Gartner 2008). ${ }^{1}$ Das Ziel von BI ist die Verbesserung des Verständnisses des Erkenntnisgegenstandes (Business) und dessen innere Zusammenhänge. Das Ziel

\footnotetext{
${ }^{1}$ Der Term BI wurde bereits 1958 bei IBM in einem sehr generellen Kontext eingeführt (Luhn 1958).
} 
wird durch qualitative und quantitative Analysemöglichkeiten erreicht (Gluchowski et al. 2008, S. 89). Bezüglich der Datenintegration, Konsolidierung und Historisierung kommt dem Data Warehouse (DWH) eine große Bedeutung zu (Inmon 2005, S. 29) und bildet die Grundlage für die weitergehende Datennutzung im Rahmen von BI. Anders als bei den bisher eingesetzten Tabellenkalkulationsprogrammen, gewährleistet es Anforderungen an die Authentisierung, Autorisierung und Datenkonsistenz (Pernul und Unland 2003, S. 428). Basis der fachlichen Umsetzung ist das multidimensionale Datenmodell, welches Objekte, deren Beziehungen und Integritätsbedingungen beschreibt und dabei unterschiedliche Betrachtungsweisen der physisch gespeicherten Daten zur Verfügung stellt (Elmasri und Navathe 2007, S. 30). Aus den vorhandenen Quellsystemen erfolgt die Datenkonsolidierung mittels des Prozesses der Extraktion, Transformation und des Ladens (ETL). Die Basis der Datenkonsolidierung stellen die beschriebenen Daten des multidimensionalen Datenmodells dar. Die Strukturierung erlaubt die Kombination von technischen, geologischen und betriebswirtschaftlichen Daten und schafft somit eine explizite Wissensbasis für die Vorauskalkulation im Tagebau. Dies wird erreicht, indem tagebauspezifische Schätzfaktoren nun explizit im Datenmodell berücksichtigt werden. Da es sich bei der Vorausplanung um Prognosedaten handelt, ist eine erhöhte Nachvollziehbarkeit mittels Soll/Ist- bzw. Soll/Soll-Vergleichen über die Zeit gegeben. Zentrales Element des Datenmodells ist der Hypercube. Dieser bildet das Vorausplanungssystem ab, das wirtschaftliche Kenngrößen wie Betriebskosten, Rückstellungen, sowie finanzmathematische Kennzahlen wie den Kapitalwert beinhaltet. Folgend ist in Abbildung 1 der Lösungsansatz zur Vorausplanung im Braunkohletagebau skizziert.

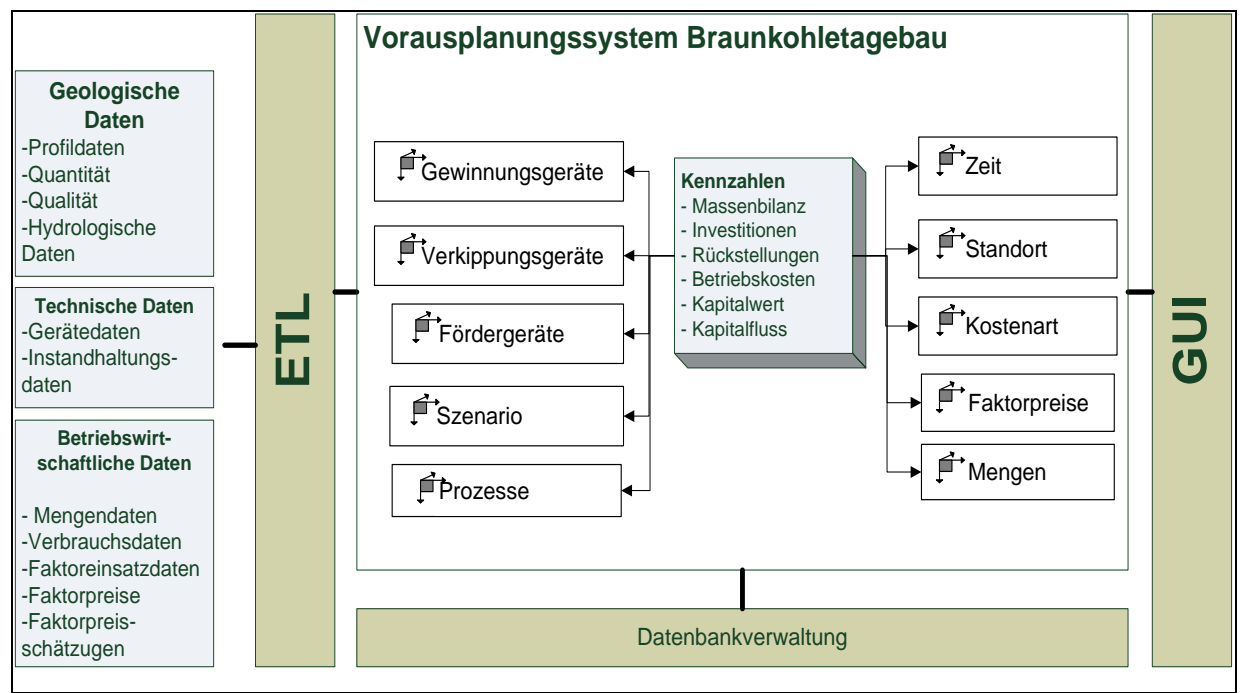

Abbildung 1: Vorausplanung im Braunkohletagebau 
Die multidimensionale Datenstruktur setzt sich aus zehn verschiedenen Dimensionen zusammen. Diese sind beispielsweise Faktorpreise, Kostenart, Mengen, Prozesse, Standort, Szenario verschiedene Gerätetypen und Zeit. Die Dimension Prozesse enthält sämtliche Parameter wie Dauer oder Produktionsfaktoren, die für den Tagebau relevant sind. Durch die Kombination mit den Dimensionen Prozesse und Faktorpreise lassen sich prozessspezifische und mengenunabhängige Kennziffern bilden. Die wesentlichen Kennzahlen wie der Kapitalwert oder der Cash-Flow sind sowohl von den Einsatzmengen bzw. Einzahlungen (Energie, Entwässerung) als auch von der Ausbringungsmenge bzw. Auszahlungen (Braunkohle) abhängig. Desweiteren ist eine Aggregation über die Zeit oder die Gliederung nach Kostenarten möglich. Der Vorteil der Datenhaltung zeigt sich in dem Vergleich der Speicherung verschiedener Soll-Szenarios. Damit erhöht sich die Nachvollziehbarkeit festgelegter Annahmen, die derzeit nicht explizit erfasst sind, z. B. Schichtfaktoren, Rekultivierungskosten oder Förderabgaben je geförderter Mengeneinheit.

\section{Forschungsrahmen und Vorgehen}

Um das Abbildungsziel eines solchen Modells zu erreichen, bedarf es einer geeigneten Methode, um zunächst die Anforderungen der Vorausplanung im Datenmodell abzubilden. Des Weiteren sollen die fachlichen Anforderungen unabhängig der physischen Implementierung sein. Während die Unabhängigkeit der fachlichen Anforderungen durch die ANSI/SPARC-Architektur (Elmasri und Navathe 2007, S. 33f) gewährleistet ist, bedarf es einem geeigneten Vorgehen zur Ermittlung der fachlichen Anforderungen im Vorausplanungsprozess.

Der Beitrag beschränkt sich auf die Darstellung des konzeptuellen Schemas der ANSI/SPARC-Architektur (Elmasri und Navathe 2007, S. 33), da dieses die fachlichen Belange der Lösung unabhängig von der Implementierung beschreibt. Der Beitrag beschränkt sich auf die Darstellung des konzeptuellen Schemas, da dieses die fachlichen Belange der Lösung unabhängig von der Implementierung beschreibt. Dabei unterscheidet das konzeptuelle Schema zwischen dem semantischen Datenmodell und dem logischen Datenmodell (Hahne 2005, S. 17). Das semantische Datenmodell dient als sprachliche Repräsentation des Fachkonzepts, während das logische Datenmodell von dem Fachkonzept abstrahiert, um eine formal-logische Implementierung zu gewährleisten (Hahne 2005, S. 16).

Um das konzeptuelle Schema zu erstellen, wird auf das Vorgehensmodell zur Erstellung eines On-Line Analytical Processing (OLAP)-Systems von Totok und Jaworski (1998, S. 26) zurückgegriffen. Das Vorgehen gliedert sich in die Anforderungsanalyse, der konzeptuellen Modellierung und der physischen Implementierung. Die Anforderungsanalyse umfasst die Untersuchung, welche qualitativen Kriterien und quantitativen Kennzahlen das System abbilden soll. Ferner sind die relevanten Datenquellen und -flüsse zu identifizieren. Die Phase der konzeptuellen Modellierung gliedert sich in die Kennzahlenbestimmung, die Ermittlung der 
Kennzahlenverknüpfungen und dem Aufbau eines Kennzahlensystems. Die gewonnen Artefakte sind Grundlage, die informationsqualifizierenden Dimensionen und die Fakten zu strukturieren, um die Kennzahlen multidimensional abzubilden.

\section{Bewertungskonzept im Bergbau}

Die fachliche Anforderungsanalyse bedarf eines Vorgehens zur Ermittlung wesentlicher finanzieller Kennzahlen im Kontext der Investitionsplanung als Grundlage der Erstellung des konzeptuellen Schemas. Zunächst erfolgt die Erläuterung der Bauwürdigkeit, um die Relevanz der Investitionsplanung zu verdeutlichen. Abschließend wird eine Bewertungsmethode einer Investitionsentscheidung im Tagebau vorgestellt, welche diejenigen Artefakte herausarbeitet, die Grundlage des zu erstellenden Datenmodells sind.

\subsection{Bauwürdigkeit einer Lagerstätte}

Die Bauwürdigkeitsprüfung ist ein kontinuierlicher Prozess, der sowohl die wirtschaftliche Bewertung von Lagerstätten als auch auf die Bewertung von Investitionen berücksichtigt, um den Erhalt der Produktionskapazitäten zu gewährleisten (Slaby und Wilke 2005, S. 22). Die Investitionsplanung bezieht sich auf Fragestellungen der Anschaffung, Weiternutzung oder des Austauschs von Betriebsmitteln (Griebel 1990, S. 266). Betriebsmittel sind alle Geräte, die zur Gewinnung und Verkippung, Förderung und Aufbereitung eingesetzt werden (Slaby und Wilke 2006, S. 166). Somit bezieht sich die Investitionsplanung auf alle Stadien eines bestimmten Tagebaus. Kritische Erfolgsfaktoren sind hierbei eine hohe Auslastung, Zeit- und Leistungsverfügbarkeit der Betriebsmittel. Deren Bewertung erfolgt aufgrund der hohen Spezifität im Tagebau anhand der Kapitalwertmethode, die den subjektiven Wert eines Gutes anhand aller mit diesem Gut zukünftig zu erzielenden Erträge darstellt (Götze 2008, S. 71). Die Dimensionierung der Fördermengen ist dabei so zu gestalten, dass ein optimales Fördermengen-Zeit-Profil erreicht wird. Dieses ist dann erreicht, wenn in jeder Zeitperiode eine Rohstoffmenge abgebaut wird, die nicht mehr Erlöse pro Mengeneinheit in einer anderen Periode erzielen könnte (Hotelling 1931, S. 140). Im Kontext der Bauwürdigkeitsprüfung basieren die betriebswirtschaftlichen Bewertungen einer Lagerstätte sowohl auf detaillierten als auch auf erfahrungsbasierenden geologischen Daten sowie einem grundlegenden technischen Abbaukonzept, woraus sich der Investitionsbedarf ergibt (Haase und Pfennig, 2009b, 131).

\subsection{Bewertungsmethode}

Die Bewertung einer Investitionsentscheidung ist von den Analyseanforderungen und dem vorliegenden Datenbestand abhängig. Um eine Bewertung effektiv und 
effizient auszuführen, bedarf es einer Methode, die ein mittelbares System von Regeln darstellt, das von den Akteuren als Handlungsplan verwendet wird (Herrman 1999, S. 37). Erste Ansätze eines formalisierten Vorgehens zur Ermittlung betriebswirtschaftlicher Kennzahlen liefern Fettweis et al. (1990). Jedoch handelt es sich hierbei kein explizites Vorgehen zur Ermittlung von Kennzahlen zur Vorausplanung im Tagebau. Daher wird als Gestaltungsgrundlage des zu erstellenden Datenmodells die dreistufige Bewertungsmethode von Slaby und Wilke (2006, S. 71) verwendet, die sämtliche Artefakte einer Investitionsentscheidung abbildet und ein Vorgehen zur Ermittlung der Kapitalflüsse, Kennzahlen und Varianten für ein gegebenes Produktionsprogramm vorschlägt. Auch andere Ansätze wie der von Haase und Pfennig (2009b, S. 132) bauen darauf auf.

\begin{tabular}{|c|c|c|}
\hline Schritt 1 & Schritt 2 & Schritt 3 \\
\hline \begin{tabular}{|l|} 
Massenbilanz \\
Gewinnung, Verkippung und \\
Förderweglängen und \\
Transportentfernung nach \\
Standorten \\
\end{tabular} & $\begin{array}{l}\text { Investitionen } \\
\text { Abschreibungen } \\
\text { Rückstellungen } \\
\text { Betriebskosten }\end{array}$ & $\begin{array}{l}\text { Gesamtkosten } \\
\text { Kapitalwert }\end{array}$ \\
\hline \begin{tabular}{|l} 
Spezifische \\
Verbräuche \\
Nach Betriebsmitteln \\
Exogene Parameter \\
Faktorpreise, Schätzfaktoren
\end{tabular} & Nach jährlichen Kohlemengen & $\begin{array}{c}\text { Kapitalflüsse } \\
\text { Variantenvergleich }\end{array}$ \\
\hline
\end{tabular}

Abbildung 2: Bewertungsmethode nach Slaby und Wilke

Die erste Stufe umfasst drei Module, die Massenbilanz, die spezifischen Kennziffern der Betriebsmittel sowie die für die Bewertung relevanten exogenen Parameter. Das erste Modul bildet die Aufstellung einer Massenbilanz, welche das nach Jahren und Betriebspunkten gegliederte Produktionsprogramm des Tagebaus enthält. Sie führt Gewinnungs- und Verkippungsmengen sowie Transportentfernungen auf. Grundlage der Bewertung sind die prognostizierten Fördermengen und die Entwicklung des Tagebaus auf Basis geologischer Profile. Im zweiten Modul werden Verbrauchskennziffern der Betriebsmittel wie Energieverbrauch für die Berechnung der variablen Betriebskosten auf Basis von Faktorpreisen und jährlichen Produktionsmengen zusammengetragen. Ziel ist dabei die Trennung von Verbrauchskennziffern und exogenen Faktorpreisen. Zunächst erfolgt die Zuordnung der Kostenarten auf die einzelnen Gerätetypen wie Bagger, Bandwagen oder Gurtbandförderer. Die Kostenarten differenzieren zwischen Investitionskosten, variablen und fixen Betriebskosten, Abschreibungen und Rückstellungen. Das dritte Modul hat zum Ziel, exogene Parameter zu identifizieren und zu bewerten. $\mathrm{Zu}$ diesen Parametern gehören Energiekosten, Dienstleistungskosten, Lohnkosten oder Steuern. Hinzu kommen implizite Schätzparameter, die bisher nicht gespeichert wurden. Die zweite Stufe umfasst die jahrweise Berechnung der Betriebskosten, Investitionen und kalkulatorischen Kosten auf Grundlage des Produktionsprogramms, der spezifischen Gerätekennziffern und der exogenen Parameter. 
Dabei werden die mengenabhängigen Produktionskosten sowie die mengen- und entfernungsabhängigen Förderkosten auf Grundlage der im ersten Schritt identifizierten Kostenarten hergeleitet. Der dritte Schritt besteht aus der Konsolidierung der berechneten Kosten zur Bildung der jährlichen Kapitalflüsse, die entweder nominal oder diskontiert betrachtet werden. Ziel der Konsolidierung ist die Ermittlung der nominalen Durchschnitts- und Gesamtkosten. Dynamische Kostenrechnungsmethoden dienen dazu, die Kapitalflüsse, diskontierte Gesamtkosten und den Kapitalwert des Tagebaus zu bestimmen. Das hier zusammengefasste Konzept zur Vorauskalkulation ist Grundlage der folgenden Erstellung des Datenmodells.

\subsection{Semantisches Datenmodell}

Die in der Anforderungsanalyse gewonnen Erkenntnisse sind Grundlage des multidimensionalen Datenmodells, welches die Investitionsplanung eines Bergbaubetriebs zeigt. Die Darstellung erfolgt auf Grundlage der Modellierungssprache Application Design for Analytical Processing Technologies (ADAPT) (Bulos 1996, S. 33). Die Sprache wurde im Kontext der multidimensionalen Modellierung gewählt, da es explizit für den konzeptionellen Entwurf von multidimensionalen Datenstrukturen entwickelt wurde. Zudem lässt sich ADAPT auch mit objektorientierten Ansätzen mit Hilfe des ADAPTed UML kombinieren (Priebe und Pernul 2001). Abbildung 3 zeigt einen Ausschnitt aus dem Datenmodell und stellt die multidimensionale Datenstruktur mit den vorhandenen Dimensionen sowie beispielhaft die Dimension Bagger in ADAPT dar.

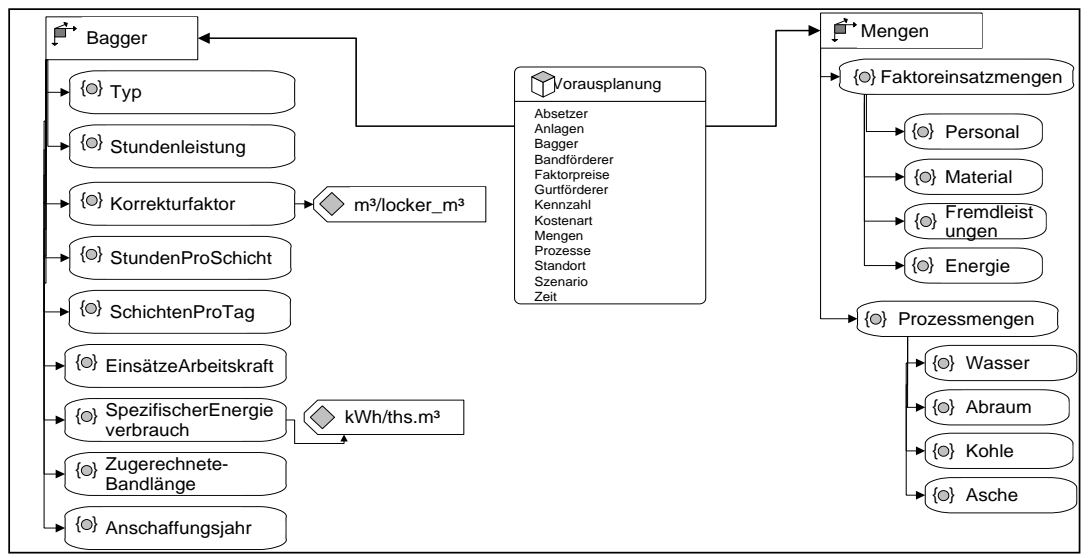

Abbildung 3: Ausschnitt des semantischen Datenmodells 


\subsection{Logische Datenmodell}

Das erstellte ADAPT-Artefakt ist Grundlage für das logische Datenmodell, welches die gewonnenen Erkenntnisse in das Star-Schema überführt, um sowohl die Fakten als auch die Dimensionen mit ihren Hierarchien, Elementen und Attributen formal zu repräsentieren. Das ADAPT Modell bildet die für die Investitionsplanung notwendigen Kennzahlen ab. Das logische Datenmodell ist die unmittelbare Grundlage der physischen Implementierung (Hahne 2005, S. 105). Die multidimensionale Struktur erlaubt es, die Artefakte der Vorauskalkulation abzubilden, gleichzeitig jedoch die bisher implizit festgelegten Schätzparameter in einer eigenen Dimensionstabelle abzubilden, um so den Vorauskalkulationsprozess personenunabhängig für verschiedene Tagebautypen durchzuführen. Abbildung 4 zeigt das Star Schema zur Massenbilanz. Die verschiedenen Gerätetypen werden aufgrund ihrer Spezifika in einer separaten Dimensionstabelle Geräte konsolidiert und anschließend mit der Faktentabelle in Beziehung gesetzt.

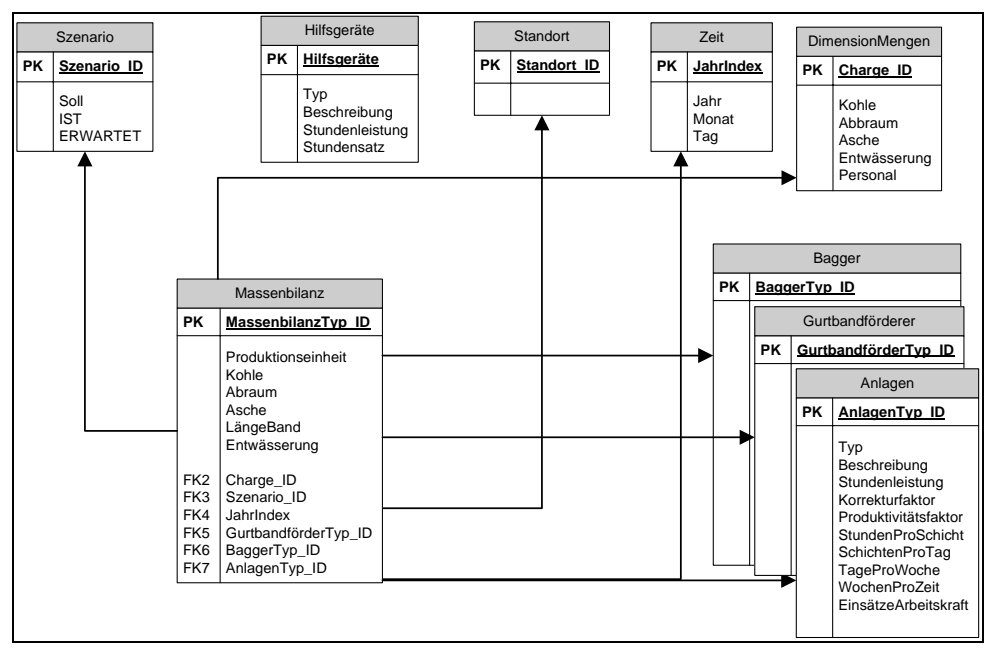

Abbildung 4: Ausschnitt logisches Datenmodell, Massenbilanz

\section{Fazit}

Ziel des Beitrages ist die Abbildung der Vorauskalkulation von Investitionsprojekten im Bergbau in einem multidimensionalen Modell, um den Vorausplanungsprozess effektiv und effizient zu unterstützen. Die Relevanz des Einsatzes einer BIgestützten Vorauskalkulation ergibt sich aus der Liberalisierung der Energiemärkte und dem einhergehenden Kostendruck. Die Braunkohle ist zwar unabhängig von der Preisbildung am Rohstoffmarkt, da Abbau und Verstromung unmittelbar zusammenhängen, jedoch zwingen gesetzliche Normen (z. B. das EnWG), die Be- 
vorzugung erneuerbarer Energien und der Handel mit $\mathrm{CO}_{2}$ Zertifikaten zu einer konsequenten Kostenplanung und -kontrolle. Daher rückt die Vorauskalkulation der Investitionen in den Vordergrund der Betrachtung, die neben betriebswirtschaftlichen Kennzahlen auch geologische und technische Artefakte berücksichtigt. Die Vorauskalkulation erfordert eine transparente Ermittlung der Plandaten sowie eine schnelle technische Bearbeitung. Die Verwendung impliziten Wissens erhöht zudem die Varianz der Planungsergebnisse aufgrund der Instabilität der Planungsparameter, so dass die Vergleichbarkeit der unterschiedlichen Planungsergebnisse erschwert wird. Andere Lösungen sind derzeit weder in der Praxis noch in der Literatur zu erkennen.

Um die genannten Nachteile des gegenwärtigen Prozesses der Vorauskalkulation zu begegnen, ist BI als geeigneter Lösungsansatz identifiziert worden. Zur Erfüllung der fachlichen Anforderungen wurde das Vorausplanungsmodell von Slaby und Wilke (2005) gewählt, um sämtliche finanzmathematischen Kennzahlen im Datenmodell abzubilden. Die fachlichen Anforderungen spiegeln sich im semantischen Datenmodell wider. Die Abbildung der Vorauskalkulation erfolgt in multidimensionalen Strukturen, um die unterschiedlichen Betrachtungsweisen der physisch gespeicherten Daten zu repräsentieren. Die multidimensionale Datenstruktur trennt zwischen den Dimensionstabellen, welche die beschreibenden Elemente der Faktentabellen enthält. Die Faktentabelle ist Basis der zuvor formulierten Kennzahlen zur Abbildung der Vorauskalkulation. Sie ist über Fremdschlüssel mit den Dimensionstabellen verknüpft. Diese Art der Verknüpfung unterstützt umfangreiche OLAP-Anforderungen, wie die mathematisch/statistische Berechnung ohne manuelle Programmierung als auch die Mehrbenutzerfähigkeit des DWH. Daher stellt das Business-Intelligence-Konzept mit seinem multidimensionalen Datenmodell in einem DWH eine Verbesserung dar, um den Prozess der Vorauskalkulation effizienter zu erfüllen und leistungsfähiger zu gestalten sowie die Nachvollziehbarkeit zu erhöhen

\section{Literatur}

Bulos D (1996) OLAP Database Design. A new dimension. Database Programming and Design 9(6): 33-37.

Elmasri R, Navathe S (2007) Fundamentals of Database Systems, 5. Auflage, Paerson International, Boston.

Felden C (2008) Regelkreisbasierter Bergtechnologischer Arbeitsplatz. In Felden C (2008) Energiewirtschaftliche Fragestellungen aus betriebswirtschaftlicher und ingenieurwissenschaftlicher Sicht, Gito, Berlin.

Fettweis GB (1990) Der Produktionsfaktor Lagerstätte. In Wahl S (Hrsg)

Bergwirtschaft Band 1, Glückauf Verlag, Essen. 
Gartner (2008) Howerd Dressner, Past Achievments http://www.gartner.com /research/fellows/asset_79427_1175.jsp.

Gluchowski P, Gabriel R, Dittmar, C (2008) Management Support Systeme und Business Intelligence, 2. Auflage, Springer, Berlin.

Götze U (2008) Investitionsrechnung: Modelle und Analysen zur Beurteilung von Investitionsvorhaben, 6. Auflage, Springer, Berlin.

Griebel HG (1990) Das Rechnungswesen im Bergbau. In Wahl S (Hrsg) Bergwirtschaft Band 1, Glückauf Verlag, Essen.

Hahne M (2005) SAP Business Information Warehouse, Springer, Berlin.

Haase B, Pfennig W (2009a) Nutzung der Braunkohle unter den Gesichtspunkten des Marktes. In Stoll RD, Niemann- Delius C, Drebenstedt C, Müllensiefen K. (2009) Der Braunkohlentagebau- Bedeutung, Planung, Betrieb, Technik, Umwelt, Springer, Berlin.

Haase B, Pfennig W (2009b) Betriebswirtschaftliche Begleitung des Prozesses der Braunkohlegewinnung. Stoll RD, Niemann- Delius C, Drebenstedt C, Müllensiefen K. (2009) Der Braunkohlentagebau- Bedeutung, Planung, Betrieb, Technik, Umwelt, Springer, Berlin.

Heck V, Weber P, Doll M (2004) Satellitengestütze Baggereinsatzplanung im Tagebau. Automatisierungstechnische Praxis 46 (11), 42-47.

Herrmann T (1999) Methoden als Problemlösungsmittel. In Holling H, Roth E (1999) Sozialwissenschaftliche Methoden, Oldenbourg, München.

Hotelling H (1931) The Economic of Exhaustible Resources. The Journal of Political Economy 39: 137-175.

Inmon W (2005) Building the Data Warehouse, 4. Auflage, Wiley Publishing, Indianapolis.

Kemper HG, Mehanna W, Unger C (2006) Business Intelligence- Grundlagen und praktische Anwendungen, 2. Auflage, Vieweg, Wiesbaden.

Konstantin P (2007) Praxishandbuch Energiewirtschaft, Springer, Berlin.

Luhn HP(1958) A Business Intelligence System. IBM Journal Oct. 1958: 314-319.

Pernul G, Unland R (2003) Datenbanken im Unternehmen- Analyse, Modellierung und Einsatz, 2. Auflage, Oldenbourg, München.

Priebe T, Pernul, G. (2001) Metadaten-gestützer Data-Warehouse-Entwurf mit ADAPTed1 UML. http://www.minet.unijena.de/sem_dwh/lit/PrPe01/.pdf 
Slaby D, Wilke FL (2005) Bergwirtschaftslehre Teil I- Wirtschaftslehre der Bergbauunternehmen und der Bergbaubetriebe, Verlag der TU Bergakademie Freiberg, Freiberg.

Slaby D, Wilke FL (2006) Bergwirtschaftslehre Teil II- Wirtschaftslehre der Bergbauunternehmen und Bergbaubetriebe. Verlag der TU Bergakademie

Totok A, Jaworski R (1998) Modellierung von multidimensionalen

Datenstrukturen mit ADAPT- Ein Fallbeispiel http://home.arcor.de/andreas. totok/pdf/Arbeitsbericht_ADAPT.pdf

Wellmer FH, Dalheimer M, Wagner M (2008) Economic Evaluations in Explorations, Springer, Berlin. 